\title{
Facile one-pot synthesis of 10-aryl derivatives of 9- aminoanthracenes and 9,10-dihydroanthracen-9-imines
}

\author{
Anlai Wang, Hongming Zhang, Sagun Tandel, Alex Black, Thomas S. Nabity, Jr., and \\ Edward R. Biehl* \\ Department of Chemistry, Southern Methodist University, Dallas, TX, USA, \\ E-mail: $\underline{\text { ebiehl@mail.smu.edu }}$
}

(received 10 Aug 00; accepted 08 Nov 01; published on the web 16 Nov 01)

\begin{abstract}
A series of 9-amino-10-arylanthracenes was easily prepared from bromobenzene and arylacetonitriles via benzyne and $\alpha$-lithiated 2-arylmethylbenzonitrile intermediates. In addition, several mono-, di-, tri-, and tetra-peri-substituted derivatives of 10-aryl-9aminoanthracenes and 9,10-dihydroanthracen-9-imines were obtained from the reaction of the respective bromoarenes as precursors of in situ generated benzyne, 3-methoxy-, 6-methoxy-3methyl-, and 3-,6-dimethoxybenzyne with preformed $\alpha$-lithiated 2-arylmethyl-benzonitriles.
\end{abstract}

Keywords: Benzyne, nucleophilic addition, amine-imine tautomerism, peri-substituted anthracene derivatives

\section{Introduction}

Substituted benzynes (generated in situ by the reaction of a bromoarene with LDA) generally react with $\alpha$-lithiated arylacetonitriles yielding the rearrangement products 2benzylbenzonitriles by a tandem addition-rearrangement pathway ${ }^{1,2}$ or $\alpha$-arylated arylacetonitriles by the usual aryne mechanism. ${ }^{3}$ However, 3,6-dimethylbenzyne 3a reacts with lithiated arylacetonitriles 4 to give 1,4,5,8-tetramethyl-9,10-dihydroanthracen-9-imines 8 (Scheme 1). ${ }^{4}$ Presumably, the imines 8 are formed by an initial non-concerted [2+2] 
cycloaddition of 3a and $\mathbf{4}$ to give the benzocyclobutene intermediate $\mathbf{5}$ which fragments to the $\alpha$-lithiated 2-benzyl-3,6-dimethylbenzonitrile 7. Usually, $\alpha$-lithiated 2-benzonitriles simply await protonation to give 2-arylbenzonitriles. However, 7 undergoes [4+2] cycloaddition with another molecule of 3a to give the observed product 8 . We have extended this reaction to include the preparation of 10-aryl derivatives of 9-amino-anthracenes and 9,10dihydroanthracen-9-imines, and report the results herein.

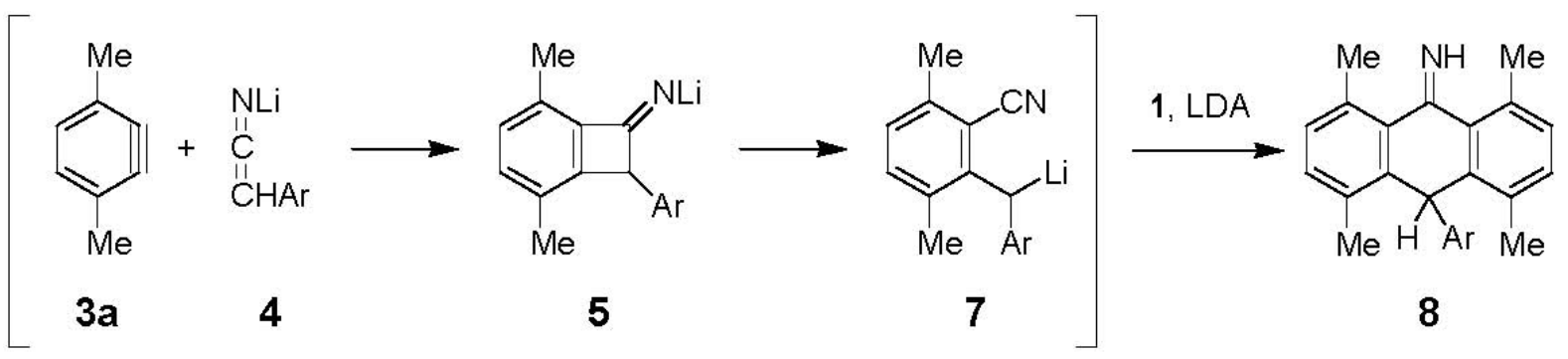

\section{Scheme 1}

Prior to this study the only reported synthesis of 9-amino-10-phenylanthracene 9a involved the successive nitration and reduction of 9-phenylanthracene which is commercially available though quite expensive. ${ }^{5}$ Furthermore, the synthesis of 9-phenylanthracene involves a multistep synthesis: ${ }^{6}$ (a) Friedel-Crafts alkylation of 10-bromo-9-anthrone with benzene to give 10-phenyl-9-anthrone; (b) reduction of phenylanthrone to 9-phenylanthracene by distillation from zinc (c) nitration of phenylanthracene to 9-nitro-10-phenylanthracene; (d) reduction of the nitro group by stannous chloride. Furthermore, the method is not general because the initial Friedel-Craft reaction is limited to benzene; using other arenes like toluene, xylenes results only in formation of resinous products.

\section{Results and Discussion}

\section{9-Amino-10-arylanthracenes}

The reaction of bromobenzene $\mathbf{1 b}$ with arylacetonitriles $\mathbf{2 a - g}$ in the presence of LDA afforded 9-amino-10-arylanthracenes $\mathbf{9 a - g} \quad(52-79 \%$ yields $)$ via benzyne $\mathbf{3 b}$ and lithiated arylacetonitriles $\mathbf{4 a - g}$ (Scheme 2). In addition, treatment of $\mathbf{1 b}$ with 3-thienylacetonitrile $\mathbf{2 h}$ 
gave the corresponding 9-amino-10-(3-thienyl)anthracene $\mathbf{9 h}$ and naphtho[2,3-b]thiophen-9amine 10 (Scheme 3). Elemental analyses, melting points (sealed, evacuated tube) and yields of compounds $\mathbf{9}$ and $\mathbf{1 0}$ are given in Table 1. These compounds are air and light sensitive and should be stored in evacuated amber containers in order to prevent decomposition.

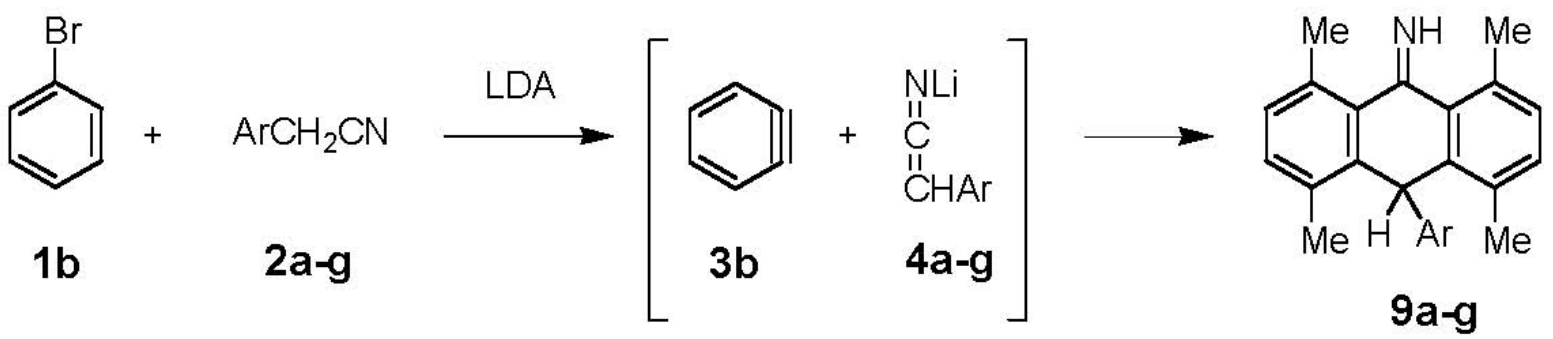

\begin{tabular}{l|l} 
& $\mathrm{Ar}$ \\
\cline { 2 - 2 } $\mathbf{a}$ & $\mathrm{C}_{6} \mathrm{H}_{5}$ \\
$\mathbf{b}$ & $4-\mathrm{MeOC}_{6} \mathrm{H}_{4}$ \\
$\mathbf{c}$ & $2-\mathrm{MeOC}_{6} \mathrm{H}_{4}$ \\
d & $3-\mathrm{MeC}_{6} \mathrm{H}_{4}$ \\
e & $1-$ naphthyl \\
f & $3,4-(\mathrm{MeO})_{2} \mathrm{C}_{6} \mathrm{H}_{3}$ \\
g & 2-pyridyl
\end{tabular}

\section{Scheme 2}

The structures of 9a-h and $\mathbf{1 0}$ were established by IR, ${ }^{1} \mathrm{H}$ NMR, ${ }^{13} \mathrm{C}$ NMR, and UV spectra. For example, the IR spectra of 9a-h exhibit two NH stretching absorption bands at about 3490 and $3410 \mathrm{~cm}^{-1}$; the ${ }^{1} \mathrm{HNMR}$ spectra reveal signals at $\delta \sim 6.6(\mathrm{NH})$ and a doublet at $\delta 8.3$. The latter signal is attributed to deshielding of the peri-protons (i.e. 1,8-protons) by the lone pair electrons of the 9-amino group.<smiles>N#CCc1ccsc1</smiles>

\section{Scheme 3}


Table 1. Yields, mp, and elemental analyses of compounds 9-11

\begin{tabular}{|c|c|c|c|c|c|}
\hline $\begin{array}{l}\text { Compound } \\
\text { (formula) }\end{array}$ & $\begin{array}{l}\text { Yield } \\
\%\end{array}$ & $\mathrm{mp}{ }^{\circ} \mathrm{C}$ & $\begin{array}{l}\mathrm{C}, \quad \% \quad \text { Found } \\
\text { (Calcd) }\end{array}$ & $\begin{array}{l}\mathrm{H}, \quad \% \quad \text { Found } \\
\text { (Calcd) }\end{array}$ & $\begin{array}{l}\mathrm{N}, \quad \% \text { Found } \\
\text { (Calcd) }\end{array}$ \\
\hline $9 a$ & 74 & $165-166^{\mathrm{a}}$ & & & \\
\hline 9b $\left(\mathrm{C}_{21} \mathrm{H}_{17} \mathrm{NO}\right)$ & 63 & $175-177$ & $84.21(84.25)$ & $5.80(5.72)$ & $4.74(4.68)$ \\
\hline 9c $\left(\mathrm{C}_{21} \mathrm{H}_{17} \mathrm{NO}\right)$ & 59 & $213-215$ & $84.29(84.25)$ & $5.68(5.72)$ & $4.62(4.68)$ \\
\hline 9d $\left(\mathrm{C}_{21} \mathrm{H}_{17} \mathrm{~N}\right)$ & 64 & $203-205$ & $88.79(89.01)$ & $6.22(6.05)$ & $4.98(4.94)$ \\
\hline $9 e\left(\mathrm{C}_{24} \mathrm{H}_{17} \mathrm{~N}\right)$ & 64 & $\begin{array}{l}167- \\
168.5\end{array}$ & $90.41(90.25)$ & $5.33(5.46)$ & $4.44(4.39)$ \\
\hline 9f $\left(\mathrm{C}_{22} \mathrm{H}_{19} \mathrm{NO}_{2}\right)$ & 79 & $240-242$ & $80.04(80.22)$ & $6.03(5.81)$ & $4.32(4.25)$ \\
\hline 9g $\left(\mathrm{C}_{19} \mathrm{H}_{14} \mathrm{~N}_{2}\right)$ & 75 & $\begin{array}{l}177.5- \\
179\end{array}$ & $84.6(84.42)$ & $5.13(5.22)$ & $10.28(10.36)$ \\
\hline 9h $\left(\mathrm{C}_{18} \mathrm{H}_{13} \mathrm{NS}\right)$ & 52 & $177-179$ & $78.80(78.51)$ & $4.84(4.76)$ & $5.04(5.09)^{\mathrm{c}}$ \\
\hline $\mathbf{1 0}\left(\mathrm{C}_{12} \mathrm{H}_{9} \mathrm{NS}\right)$ & 31 & $122-124$ & $72.40(72.33)$ & $4.59(4.55)$ & $7.09(7.03)^{d}$ \\
\hline $11 \mathrm{a}$ & 90 & $262-263^{b}$ & & & \\
\hline $\mathbf{1 1 b}\left(\mathrm{C}_{23} \mathrm{H}_{19} \mathrm{NO}_{2}\right)$ & 83 & $274-276$ & $80.93(80.92)$ & $5.69(5.61)$ & $4.18(4.10)$ \\
\hline 11c $\left(\mathrm{C}_{23} \mathrm{H}_{19} \mathrm{NO}_{2}\right)$ & 85 & $229-231$ & 80.99 (80.92) & $5.60(5.61)$ & $4.13(4.10)$ \\
\hline 11d $\left.\mathrm{C}_{23} \mathrm{H}_{19} \mathrm{NO}\right)$ & 62 & $233-235$ & $84.77(84.89)$ & $5.97(5.89)$ & $4.37(4.30)$ \\
\hline 11e $\left(\mathrm{C}_{26} \mathrm{H}_{19}\right) \mathrm{NO}$ & 86 & $327-329$ & $86.44(86.40)$ & $5.43(5.30)$ & $3.95(3.88)$ \\
\hline $11 f\left(\mathrm{C}_{24} \mathrm{H}_{21} \mathrm{NO}_{3}\right)$ & 64 & $310-312$ & $77.65(77.61)$ & $5.78(5.70)$ & $3.87(3.77)$ \\
\hline $11 g\left(\mathrm{C}_{20} \mathrm{H}_{15} \mathrm{NOS}\right)$ & 74 & $287-289$ & $75.75(75.68)$ & $4.50(4.76)$ & $4.43(4.41)$ \\
\hline
\end{tabular}

${ }^{\mathrm{a}} \mathrm{lit}^{4} \mathrm{mp} 162-163{ }^{\circ} \mathrm{C} .{ }^{\mathrm{b}}$ lit. $^{4} \mathrm{mp} 261^{\circ} \mathrm{C} .{ }^{\mathrm{c}} \% \mathrm{~S}: 11.70$ (11.61). ${ }^{\mathrm{d}} \% \mathrm{~S}=16.21$ (16.09).

Subsequently, we found that compounds 9a-f,h can be easily converted into air-stable $\mathrm{N}$ acyl derivatives 11a-f,h (62-90\% yields) by treatment with acetic anhydride at $0{ }^{\circ} \mathrm{C}$ (Table 1). Although these amides 11a-f,h are stable in dimethylsulfoxide solution, they undergo partial tautomerization to the corresponding 9,10-dihydroanthracen-9-imine 12a-f,h in deuterochloroform (eq. 1). 


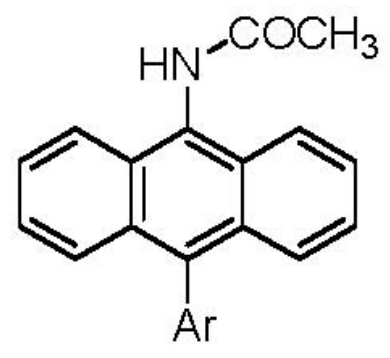

11a-f, h

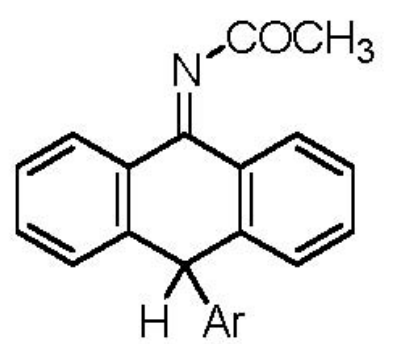

12a-f, $h$

The ${ }^{1} \mathrm{H}$ NMR and ${ }^{13} \mathrm{C}$ NMR spectra of 11a-f,h in DMSO- $d_{6}$ reveal characteristic signals in the range of $\delta 10.2-10.3(\mathrm{NH}), \delta \sim 8.13(1,8-\mathrm{H})$, and $\delta \sim 170(\mathrm{C}=\mathrm{O})$, respectively. However, the ${ }^{1} \mathrm{H}$ NMR and ${ }^{13} \mathrm{C}$ NMR spectra of $\mathrm{CDCl}_{3}$ solutions of 11a-f,h reveal the additional presence of 12a-f,h. The ${ }^{1} \mathrm{H}$ NMR spectra of $\mathrm{CDCl}_{3}$ solutions show two signals for the methyl protons of the acetyl group at $\delta 2.5$ and $\delta \sim 1.7$, two sets of doublets for the peri protons at $\delta \sim$ 7.9 and $\delta \sim 9.1$, and a considerably broadened amide proton signal as well as a broadened signal in the range of $\delta 5.5-6.0$ due to 5 - $\mathrm{H}$ of the imino tautomers. The ${ }^{13} \mathrm{C}$ NMR spectra in $\mathrm{CDCl}_{3}$ exhibit two methyl signals at $\delta 20.6$ and 23.5 and two carbonyl signals at $\delta 170.2$ and 174.6. The strong preference for the amide tautomers in dimethylsulfoxide solutions probably reflects the hydrogen bonding interactions between the solvent and the 9-amide hydrogen atom. Such interactions, of course, are absent in the imine tautomers.

\section{peri-Substituted 9-amino-10-arylanthracenes}

Furthermore, we prepared 1-methoxy- 9i-l and 1-methoxy-4-methyl-substituted derivatives 9o-s of 9-amino-10-arylanthracenes by generating benzyne $3 \mathbf{b}$ from $\mathbf{1 b}$ in the presence of $\alpha$ lithiated 6-methoxy- 7a-d and 6-methoxy-3-methyl-substituted 2-arylmethylbenzonitriles 7e-i (Scheme 3 and Table 2). The intermediates 7a-h were prepared from the corresponding benzonitriles 6a- $\mathbf{h}$ which, in turn, were available from other studies. ${ }^{7-9}$ In addition, 3methoxybenzyne 3c reacted with $\mathbf{7 a}$ and $\mathbf{7 d}$ to give the respective 10-phenyl- $\mathbf{9 m}$ and 10 (naphth-1-yl)-substituted 9n derivatives of 9-amino-1,8-dimethoxyanthracene. The reaction of 3,4,5-trimethoxybenzonitrile 6i with $\mathbf{1 d}$ gave a mixture of the 9-amino derivative 9s and 9imino derivative 8a. These tautomers were readily separated by column chromatography on silica gel using hexane/ethyl acetate (4:1) as eluent. 1,4,5,8-Tetra-substituted 9,10dihydroanthracen-9-imines $\mathbf{8 b}$-d were obtained from the reaction of the appropriate 
benzonitriles 6i,e,f with $\mathbf{1 d}$ or $\mathbf{1 e}$, respectively.

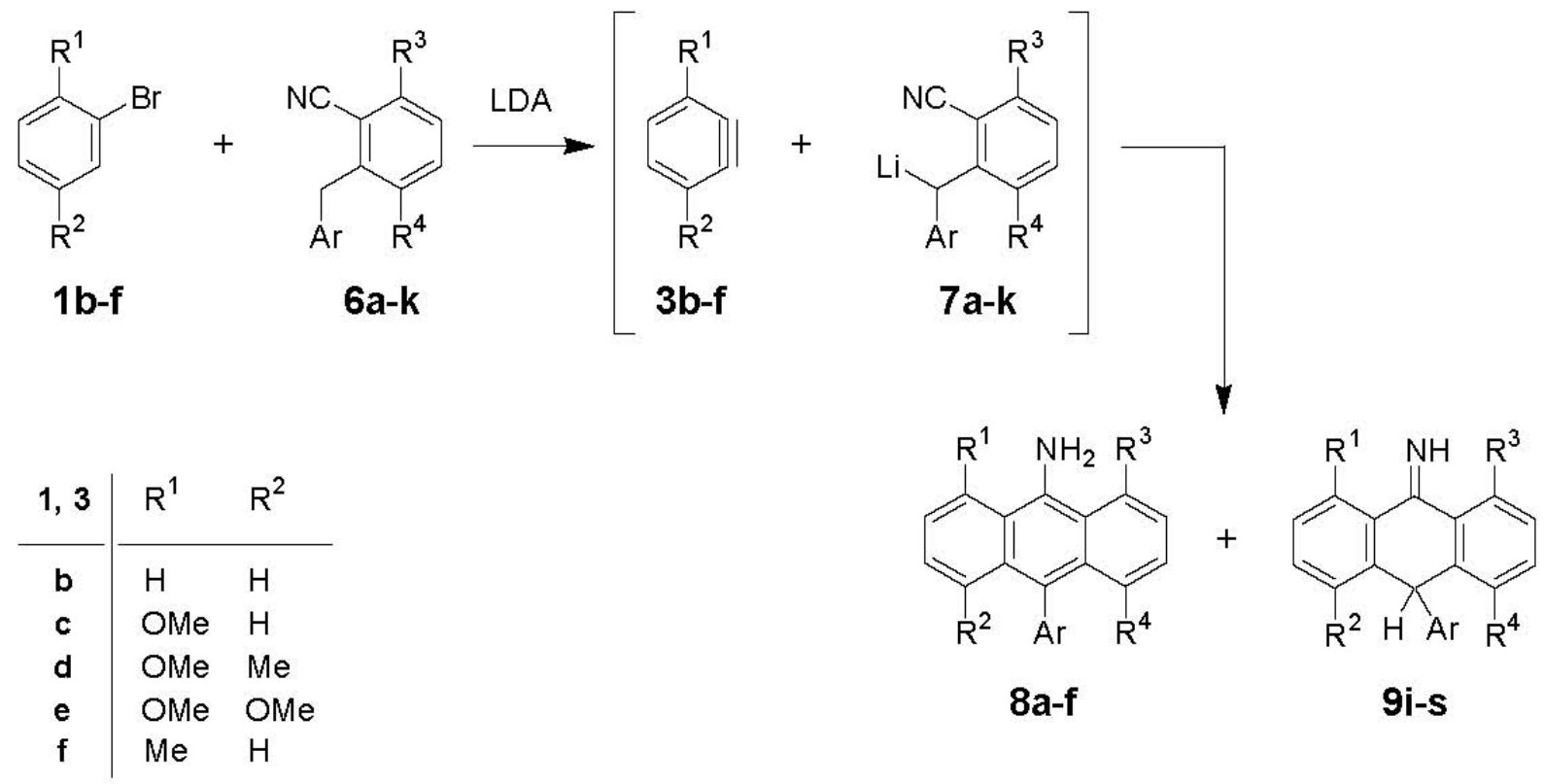

\begin{tabular}{|c|c|c|c|}
\hline 6,7 & $\mathrm{R}^{3}$ & $\mathrm{R}^{4}$ & $\mathrm{Ar}$ \\
\hline a & OMe & $\mathrm{H}$ & $\mathrm{Ph}$ \\
\hline b & OMe & $\mathrm{H}$ & 4- $\mathrm{MeOC}_{6} \mathrm{H}_{4}$ \\
\hline c & OMe & $\mathrm{H}$ & $3-\mathrm{MeC}_{6} \mathrm{H}_{4}$ \\
\hline d & OMe & $\mathrm{H}$ & 1-naphthyl \\
\hline e & OMe & $\mathrm{Me}$ & $\mathrm{Ph}$ \\
\hline$f$ & OMe & $\mathrm{Me}$ & 4- $\mathrm{MeOC}_{6} \mathrm{H}_{4}$ \\
\hline $\mathbf{g}$ & OMe & $\mathrm{Me}$ & $3-\mathrm{MeC}_{6} \mathrm{H}_{4}$ \\
\hline $\mathrm{h}$ & OMe & $\mathrm{Me}$ & 1-naphthyl \\
\hline $\mathbf{i}$ & OMe & $\mathrm{Me}$ & $3,4,5-(\mathrm{MeO})_{3} \mathrm{C}_{6} \mathrm{H}_{2}$ \\
\hline j & $\mathrm{Me}$ & $\mathrm{H}$ & 2- $\mathrm{MeOC}_{6} \mathrm{H}_{4}$ \\
\hline k & $\mathrm{H}$ & $\mathrm{Me}$ & 2- $\mathrm{MeOC}_{6} \mathrm{H}_{4}$ \\
\hline
\end{tabular}

\begin{tabular}{|c|c|c|c|c|c|}
\hline 8 & $\mathrm{R}^{1}$ & $\mathrm{R}^{2}$ & $\mathrm{R}^{3}$ & $\mathrm{R}^{4}$ & $\mathrm{Ar}$ \\
\hline a & $\mathrm{H}$ & $\mathrm{H}$ & OMe & $\mathrm{Me}$ & $3,4,5-(\mathrm{MeO})_{3} \mathrm{C}_{6} \mathrm{H}_{2}$ \\
\hline b & OMe & $\mathrm{Me}$ & OMe & $\mathrm{Me}$ & 4- $\mathrm{MeOC}_{6} \mathrm{H}_{4}$ \\
\hline c & OMe & OMe & OMe & $\mathrm{Me}$ & $\mathrm{Ph}$ \\
\hline d & OMe & $\mathrm{H}$ & OMe & $\mathrm{Me}$ & 4- $\mathrm{MeOC}_{6} \mathrm{H}_{4}$ \\
\hline e & $\mathrm{H}$ & $\mathrm{Me}$ & $\mathrm{Me}$ & $\mathrm{H}$ & $2-\mathrm{MeOC}_{6} \mathrm{H}_{4}$ \\
\hline f & $\mathrm{Me}$ & $\mathrm{H}$ & OMe & $\mathrm{Me}$ & 4- $\mathrm{MeOC}_{6} \mathrm{H}_{4}$ \\
\hline 9 & $\mathrm{R}^{1}$ & $\mathrm{R}^{2}$ & $\mathrm{R}^{3}$ & $\mathrm{R}^{4}$ & $\mathrm{Ar}$ \\
\hline $\mathbf{i}$ & $\mathrm{H}$ & $\mathrm{H}$ & OMe & $\mathrm{H}$ & $\mathrm{Ph}$ \\
\hline j & $\mathrm{H}$ & $\mathrm{H}$ & OMe & $\mathrm{H}$ & 4- $\mathrm{MeOC}_{6} \mathrm{H}_{4}$ \\
\hline k & $\mathrm{H}$ & $\mathrm{H}$ & OMe & $\mathrm{H}$ & $3-\mathrm{MeC}_{6} \mathrm{H}_{4}$ \\
\hline I & $\mathrm{H}$ & $\mathrm{H}$ & OMe & $\mathrm{H}$ & 1-naphthyl \\
\hline m & OMe & $\mathrm{H}$ & OMe & $\mathrm{H}$ & $\mathrm{Ph}$ \\
\hline $\mathbf{n}$ & OMe & $\mathrm{H}$ & OMe & $\mathrm{H}$ & 1-naphthyl \\
\hline 0 & $\mathrm{H}$ & $\mathrm{H}$ & OMe & $\mathrm{Me}$ & $\mathrm{Ph}$ \\
\hline $\mathbf{p}$ & $\mathrm{H}$ & $\mathrm{H}$ & OMe & $\mathrm{Me}$ & 4- $\mathrm{MeOC}_{6} \mathrm{H}_{4}$ \\
\hline$q$ & $\mathrm{H}$ & $\mathrm{H}$ & OMe & $\mathrm{Me}$ & $3-\mathrm{MeC}_{6} \mathrm{H}_{4}$ \\
\hline $\mathbf{r}$ & $\mathrm{H}$ & $\mathrm{H}$ & OMe & $\mathrm{Me}$ & 1-naphthyl \\
\hline $\mathbf{s}$ & $\mathrm{H}$ & $\mathrm{H}$ & OMe & $\mathrm{Me}$ & $3,4,5-(\mathrm{MeO})_{3} \mathrm{C}_{6} \mathrm{H}_{2}$ \\
\hline
\end{tabular}

\section{Scheme 4}


The products were identified on the basis of IR, ${ }^{1} \mathrm{H}$ NMR, ${ }^{13} \mathrm{C}$ NMR and UV spectra. The UV spectra of the bright yellow 9-aminoanthracene derivatives exhibit characteristic absoprtion bands at $\lambda_{\max } 420,380,340 \mathrm{~nm}$. The ${ }^{1} \mathrm{H}$ NMR spectra of 9i-9s display characteristic downfield shifts $(\delta \sim 8.1)$ and coupling patterns (doublet of doublet) characteristic of peri protons (resulting from ${ }^{3} J$ and ${ }^{4} J$ ). These coupling patterns are absent in the ${ }^{1} \mathrm{H}$ NMR spectra of 1,8-dimethoxy-substituted 9-aminoanthracenes $\mathbf{9 m}$ and $\mathbf{9 n}$. Interestingly, ${ }^{1} \mathrm{H}$ NMR spectra of these two aminoanthracenes $\mathbf{9 m}$ and $\mathbf{9 n}$ are particularly revealing: the signals of the 9 -amino protons occur at $\delta 8.10$ and 8.26 , respectively. These signals are considerably shifted downfield (approximately 1.5-1.75 ppm) as compared to those of the other 9-aminoanthracenes reported in this study. This shift probably reflects extended intramolecular H-bonding between the two amino hydrogen atoms and the adjacent 1,8-dimethoxy substituents. On the other hand, the ${ }^{1} \mathrm{H}$ NMR spectra of the imines 8a-d exhibit a characteristic NH signal at $\delta \sim 11.0$, and a 10 - $\mathrm{H}$ singlet at $\delta 5.5$, whereas the ${ }^{13} \mathrm{C}$ NMR spectra show characteristic $\mathrm{C}=\mathrm{N}$ signals at $\delta \sim 186$.

We were also able to introduce methyl group/s into the anthracene ring using 2bromotoluene 1f as aryne precursor in two ways. First, 1f was treated with (2methoxyphenyl)acetonitrile 2c and LDA to yield a 1:1 mixture (determined by GC/MS and by integration of the respective aromatic hydrogen signals of each regioisomer) of lithiated 2-(2methoxyphenylmethyl)-6-methylbenzonitrile $\quad 7 \mathbf{j}$ and 2-(2-methoxy-phenylmethyl)-3methylbenzonitrile 7k (Scheme 5). This is consistent with the weak inductive effect of the methyl group in 3-methylbenzyne. ${ }^{10}$ No attempt was made to separate the mixture, it rather was made to react with 2-bromotoluene $1 f$ and LDA to give a single product, 10-(2-methoxyphenyl)-1,5-dimethyl-9,10dihydroanthracen-9-imine 8e (62\% yield); none of the 1,8- or 1,5dimethyl regioisomers was detected. The structure of $\mathbf{8 e}$ was confirmed by single crystal XRay spectrometry, the ORTEP ${ }^{7}$ drawing is shown in Figure 1. 


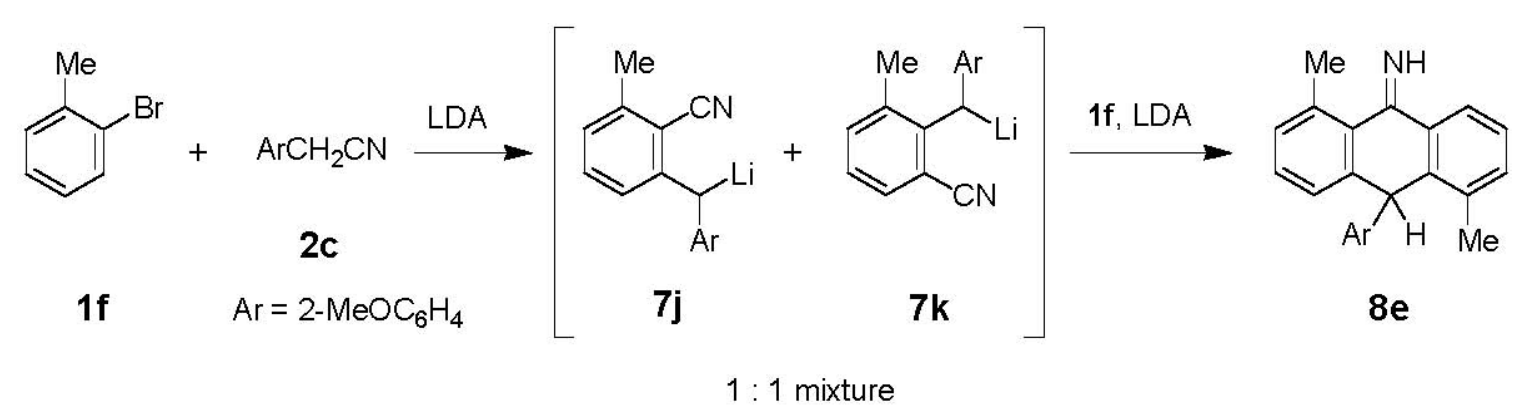

\section{Scheme 5}

Most likely, the exclusive formation of $\mathbf{8} \mathbf{e}$ involves the regioselective addition of $\alpha$ lithiated 5-methylbenzonitrile $\mathbf{7} \mathbf{j}$ to the 2-position of the aryne $\mathbf{3} \mathbf{f}$ and the opposite addition of $\mathbf{7 k}$ to the 3-position of $\mathbf{3} \mathbf{f}$ (Scheme 5). The regioselectve formation of $\mathbf{8 e}$ from both isomeric $\alpha$-lithiated nitrile intermediates $\mathbf{7} \mathbf{j}$ and $\mathbf{7 k}$ is caused by steric effects. Obviously, the carbanion $7 \mathbf{j}$ adds exclusively to the 2-position of 3-methylbenzyne $3 \mathbf{f}$, whereas the carbanion $\mathbf{7 k}$ adds to the 3-position of 3f. The opposite regioselective additions by the two isomeric nucleophiles $\mathbf{7 j}$ and can not be explained on the basis of the inductive effect of the methyl (which is weak) but rather on the steric effect of the methyl. Inspection of molecular models reveals that steric interactions between the methyl groups and the 9-imino and 10-aryl group in $\mathbf{8 e}$ are less severe than those in which the two methyl groups flank either the 9-imino or the 10-aryl group. Consequently, the tricyclic ring in $\mathbf{8 e}$ is not strongly puckered, and hence, it is more stabilized by resonance as compared to the situation of the other two possible isomers.

Table 2. Yields, mp, and elemental analyses of compounds 9i-9s and 8a-f

\begin{tabular}{|c|c|c|c|c|c|}
\hline $\begin{array}{l}\text { Compound } \\
\text { (formula) }\end{array}$ & $\begin{array}{l}\text { Yield } \\
(\%)\end{array}$ & $m p{ }^{\circ} \mathrm{C}$ & $\begin{array}{lr}\text { C, \% } & \text { Found } \\
\text { (Calcd) } & \\
\end{array}$ & 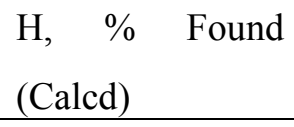 & $\begin{array}{lll}\mathrm{N}, \quad \% & \text { Found } \\
(\mathrm{Calcd}) & \end{array}$ \\
\hline $9 \mathbf{i}\left(\mathrm{C}_{21} \mathrm{H}_{17} \mathrm{NO}\right)$ & 57 & $\begin{array}{l}175- \\
177\end{array}$ & $84.31(84.25)$ & $5.77(5.72)$ & $4.60(4.68)$ \\
\hline $\mathbf{9 j}\left(\mathrm{C}_{22} \mathrm{H}_{19} \mathrm{NO}_{2}\right)$ & 52 & $\begin{array}{l}136- \\
139\end{array}$ & $80.27(80.22)$ & $5.88(5.82)$ & $4.27(4.25)$ \\
\hline 9k $\left(\mathrm{C}_{21} \mathrm{H}_{17} \mathrm{NO}\right)$ & 32 & $\begin{array}{l}157- \\
159 \\
\end{array}$ & $84.40(84.31)$ & $6.15(6.11)$ & $4.50(4.47)$ \\
\hline
\end{tabular}


Table 2. Continued

\begin{tabular}{|c|c|c|c|c|c|}
\hline $9 \mathbf{l}\left(\mathrm{C}_{25} \mathrm{H}_{19} \mathrm{NO}\right)$ & 48 & $\begin{array}{l}152- \\
155\end{array}$ & $85.88(85.92)$ & $5.53(5.48)$ & $4.05(4.01)$ \\
\hline $9 \mathbf{m}\left(\mathrm{C}_{22} \mathrm{H}_{19} \mathrm{NO}_{2}\right)$ & 63 & $\begin{array}{l}157- \\
159\end{array}$ & $80.29(80.22)$ & $5.87(5.81)$ & $4.22(4.25)$ \\
\hline 9n $\left(\mathrm{C}_{26} \mathrm{H}_{21} \mathrm{NO}_{2}\right)$ & 38 & $\begin{array}{l}214- \\
216\end{array}$ & $82.37(82.30)$ & $5.59(5.58)$ & $3.72(3.69)$ \\
\hline $9 \mathbf{o}\left(\mathrm{C}_{22} \mathrm{H}_{19} \mathrm{NO}\right)$ & 35 & $\begin{array}{l}147- \\
149\end{array}$ & $84.38(84.31)$ & $6.15(6.11)$ & $4.54(4.47)$ \\
\hline $9 \mathbf{p}\left(\mathrm{C}_{23} \mathrm{H}_{21} \mathrm{NO}_{2}\right)$ & 68 & $\begin{array}{l}212- \\
214\end{array}$ & $84.45(84.44)$ & $6.11(6.16)$ & $4.14(4.08)$ \\
\hline $9 \mathbf{q}\left(\mathrm{C}_{23} \mathrm{H}_{21} \mathrm{NO}\right.$ & 64 & $\begin{array}{l}204- \\
205\end{array}$ & $84.42(84.37)$ & $6.49(6.46)$ & $4.31(4.28)$ \\
\hline 9r $\left(\mathrm{C}_{26} \mathrm{H}_{21} \mathrm{NO}\right.$ & 51 & $\begin{array}{l}188- \\
190\end{array}$ & $86.01(85.92)$ & $5.88(5.82)$ & $3.88(3.85)$ \\
\hline 9s $\left(\mathrm{C}_{25} \mathrm{H}_{25} \mathrm{NO}_{4}\right)$ & 43 & $\begin{array}{l}106- \\
108\end{array}$ & $74.38(74.42)$ & $6.28(6.25)$ & $3.55(3.47)$ \\
\hline $\mathbf{8 a}\left(\mathrm{C}_{25} \mathrm{H}_{25} \mathrm{NO}_{4}\right)$ & 21 & $\begin{array}{l}178- \\
180\end{array}$ & $74.50(74.42)$ & $6.32(6.25)$ & $3.52(3.47)$ \\
\hline $\left.\mathbf{8 b} \mathrm{C}_{25} \mathrm{H}_{25} \mathrm{NO}_{3}\right)$ & 68 & $\begin{array}{l}221- \\
223\end{array}$ & 77.55 (77.49) & $6.62(6.50)$ & $3.66(3.61)$ \\
\hline $\mathbf{8 c}\left(\mathrm{C}_{24} \mathrm{H}_{23} \mathrm{NO}_{3}\right)$ & 47 & $\begin{array}{l}182- \\
183\end{array}$ & 77.25 (77.19) & $6.16(6.21)$ & $3.77(3.75)$ \\
\hline $\mathbf{8 d}\left(\mathrm{C}_{23} \mathrm{H}_{21} \mathrm{NO}_{2}\right)$ & 54 & $\begin{array}{l}191- \\
193\end{array}$ & $80.46(80.44)$ & $6.22(6.16)$ & $4.11(4.08)$ \\
\hline $\mathbf{8 e}\left(\mathrm{C}_{23} \mathrm{H}_{21} \mathrm{NO}\right)$ & 62 & $\begin{array}{l}180- \\
182\end{array}$ & 84.43 (84.37) & $6.70(6.46)$ & $4.32(4.28)$ \\
\hline $\mathbf{8 f}\left(\mathrm{C}_{24} \mathrm{H}_{23} \mathrm{NO}_{2}\right)$ & 57 & $\begin{array}{l}177- \\
179\end{array}$ & $80.70(80.64)$ & $6.44(6.49)$ & $3.99(3.92)$ \\
\hline
\end{tabular}




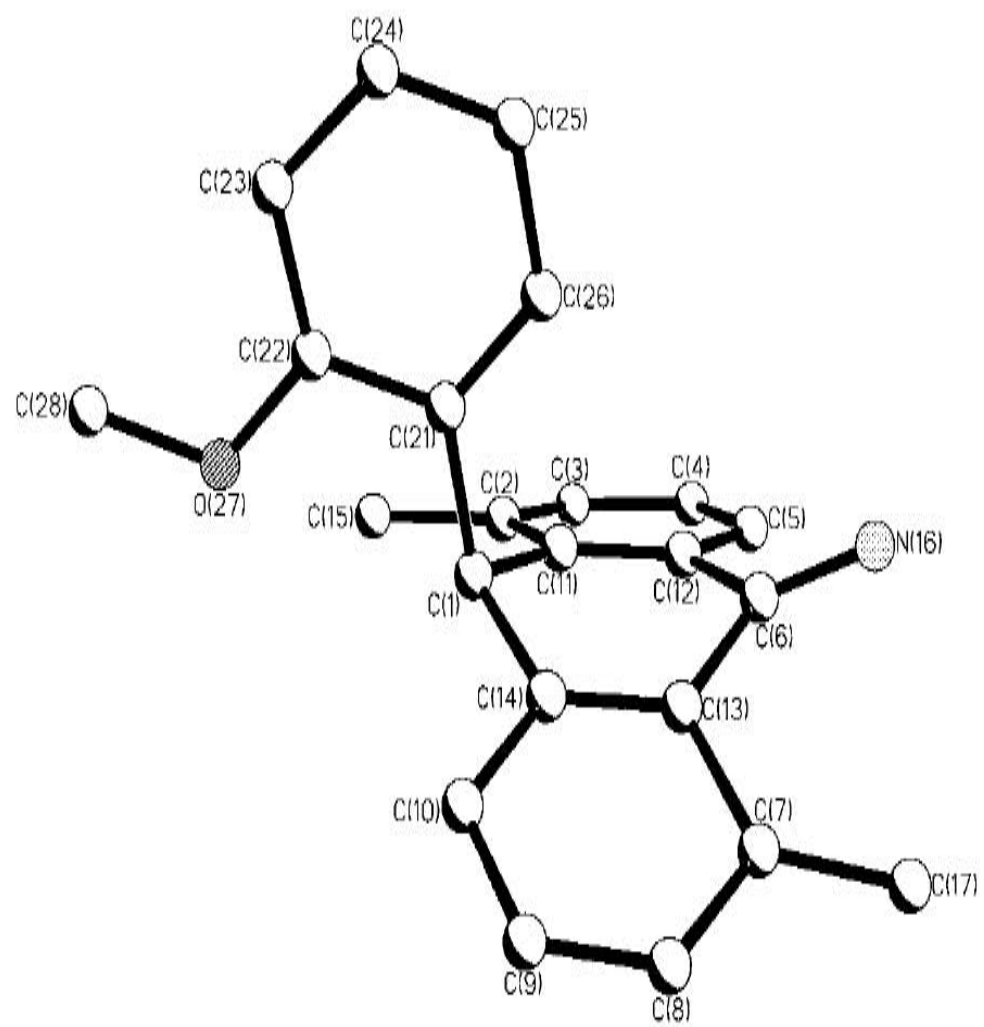

Figure 1. ORTEP drawing of $\mathbf{8 e}$<smiles>Cc1ccccc1</smiles><smiles>Cc1ccccc1</smiles><smiles>CC</smiles><smiles>Cc1cccc2c1C(=N)c1cccc(C)c1C2Br</smiles>

$8 e$

$7 k$

$3 f$

Scheme 6 
Similarly, the reaction of bromotoluene 1f with $\mathbf{6 f}$ and LDA gave a single product, 1methoxy-10-(4-methoxyphenyl)-4,8-dimethyl-9,10-dihydroanthracen-9-imine 8f (57\% yield) (eq 2). Again, nucleophilic addition occurred in such a way to place the two methyl groups on opposite sides of the tricyclic ring.

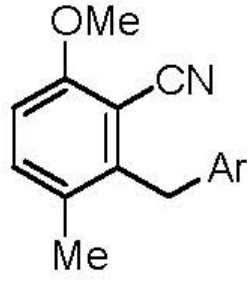

$6 f$

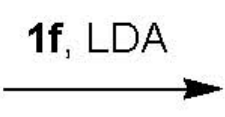

$8 \mathrm{e}$ eq. 2

$$
\mathrm{Ar}=2-\mathrm{MeOC}_{6} \mathrm{H}_{4}
$$

In conclusion, the otherwise not easily accessible 10-aryl-substituted derivatives of 9aminoanthacenes 9 and 9,10-dihydroanthracen-9-imines 8 are readily prepared by the one-pot synthesis described herein. Utilizing readily available and inexpensive starting materials provides an efficient access to these products in multi-gram quantities.

\section{Experimental Section}

General Procedures. Melting points were taken on a Mel-Temp II capillary apparatus, and are uncorrected with respect to stem correction. IR spectra were recorded on a Nicolet Magna$\mathrm{IR}^{\mathrm{TM}} 550$ FTIR spectrometer and the ${ }^{1} \mathrm{H}$ and ${ }^{13} \mathrm{C}$ NMR spectra were recorded on a $400 \mathrm{MHz}$ Bruker AVANCE DRX-400 Multi-nuclear NMR spectrometer; chemical shifts were referenced to TMS as internal standard. The UV/VIS spectra were recorded on a Beckman DU 660 Spectrometer. Elemental analyses were obtained from SMU Analytical Services Laboratories.

Bromobenzene, arylacetonitriles, diisopropylamine, 2,2,6,6-tetramethylpiperidine, and $n$ BuLi were purchased from Aldrich Chemical Company. Diisopropylamine and 2,2,6,6tetramethylpiperidine were refluxed over and distilled from calcium hydride. Tetrahydrofuran (THF) was distilled from Na/benzophenone immediately prior to use. The glassware was 
heated at $125{ }^{\circ} \mathrm{C}$ in an oven overnight prior to use. Benzyne reactions were carried out under an atmosphere of dry $\mathrm{O}_{2}$-free $\mathrm{N}_{2}$ utilizing a balloon.

\section{General procedure for the preparation of 10-aryl-9-aminoanthracenes 9a-h}

The reactions and the work-up were carried out in subdued light. Fresh LDA (40 mmol) was prepared in a flame-dried flask flushed with nitrogen by adding $n$-BuLi (40 mmol, $2.5 \mathrm{M}$ in hexane) to a solution of diisopropylamine $(4.0 \mathrm{~g}, 40 \mathrm{mmol})$ in $\mathrm{THF}(50 \mathrm{~mL})$ at $-70^{\circ} \mathrm{C}$. After stirring for $10 \mathrm{~min}$, the appropriate arylacetonitrile $2(10 \mathrm{mmol})$ was added, and stirring was contiuned for $20 \mathrm{~min}$ to ensure complete anion formation. Bromobenzene $\mathbf{1 b}(0.312 \mathrm{~g}, 20$ mmol) was added, the resulting solution was allowed to warm to room temperature and was stirred for $6 \mathrm{~h}$. The reaction was then quenched with saturated $\mathrm{NH}_{4} \mathrm{Cl}$ solution $(30 \mathrm{~mL})$ and extracted with methylene chloride. The combined extracts were washed with dilute $\mathrm{HCl}$ then dried $\left(\mathrm{Na}_{2} \mathrm{SO}_{4}\right)$ and concentrated (rotary evaporator) to give a crude material. Chromatography of this material on silica gel (hexane/ethyl acetate, 4:1) gave the pure product 9a-h. The yields, mp and elemental analyses for 9a-h are shown in Table 1 and spectral data of 12ag are given below.

9-Amino-10-phenylanthracene (9a). IR (KBr): $v_{\max }$ 3487, $3407 \mathrm{~cm}^{-1}\left(\mathrm{NH}_{2}\right)$; UV $\left(\mathrm{CHCl}_{3}\right)$ : $\lambda_{\max }[\mathrm{nm}](\log \varepsilon): 272$ (5.07) [lit. $\left.{ }^{5} 2.72\{5.07\}\right], 360$ (3.79), [lit. $\left.{ }^{5} 381\{3.74\}\right] 420$ (4.00) [lit. ${ }^{5}$ $420\{4.00\}] ;{ }^{1} \mathrm{H}$ NMR (DMSO-d 6 ): $\delta 6.60$ (s, 2H), 7.32-7.34 (m, 2H), 7.40-7.42 (m, 5H), 7.51-7.57 (m, 4H), $8.33(\mathrm{~d}, J=8.8 \mathrm{~Hz}, 2 \mathrm{H}) ;{ }^{13} \mathrm{C} \mathrm{NMR}\left(\mathrm{CDCl}_{3}\right): \delta 117.9,121.1,123.7,125.3$, 125.9, 127.1, 127.4, 128.4, 130.6, 132.1, 137.9, 139.6

9-Amino-10-(4-methoxyphenyl)anthracene (9b). IR (KBr): $v_{\max } 3474,3388 \mathrm{~cm}^{-1}\left(\mathrm{NH}_{2}\right)$; UV $\left(\mathrm{CHCl}_{3}\right): \lambda_{\max }[\mathrm{nm}](\log \varepsilon): 270$ (4.59), 360 (3.55), 380 (3.73), 420 (3.78); NMR (DMSO$\left.d_{6}\right): \delta 3.95(\mathrm{~s}, 3 \mathrm{H}), 6.64(\mathrm{~s}, 2 \mathrm{H}), 7.09,7.11\left(\mathrm{AA}^{\prime}\right.$ of 4-MeOC $\left.\mathrm{H}_{4}\right), 7.27,7.29\left(\mathrm{BB}^{\prime}\right), 7, .31-7.35$ $(\mathrm{m}, 2 \mathrm{H}), 7.42(\mathrm{~m}, 2 \mathrm{H}), 7.44(\mathrm{~d}, J-=8.8 \mathrm{~Hz}, 2 \mathrm{H}), 8.38(\mathrm{~d}, J=8.8 \mathrm{~Hz}, 2 \mathrm{H}) ;{ }^{13} \mathrm{C} \mathrm{NMR}\left(\mathrm{CDCl}_{3}\right)$ : $\delta 55.4,113.9,117.9,121.1,123.7,125.2,126.9,127.2,127.7,130.9,131.6,133.1,137.8$, 158.8 .

9-Amino-10-(2-methoxyphenyl)anthracene (9c). IR (KBr): $v_{\max } 3490,3403,1665 \mathrm{~cm}^{-1}$ $\left(\mathrm{NH}_{2}\right)$; UV: $\left(\mathrm{CHCl}_{3}\right) \lambda_{\max }[\mathrm{nm}](\log \varepsilon): 269$ (4.91), 360 (3.55), 3.80 (3.87), $420(3.91) ;{ }^{1} \mathrm{H}$ NMR (DMSO-d $\left.d_{6}\right): \delta 3.57$ (s, 3H), 6.60 (s, 2 H), 7.12-7.13 (m, 2 H), 7.19-7.22 (m, 2 H), 7.24$7.30(\mathrm{~m}, 6 \mathrm{H}), 7.48-7.49(\mathrm{~m}, 1 \mathrm{H}), 8.32(\mathrm{~d}, J=8.8 \mathrm{~Hz}, 2 \mathrm{H}) ;{ }^{13} \mathrm{C} \mathrm{NMR}\left(\mathrm{CDCl}_{3}\right): \delta 55.8$, 
$111.4,118.1,120.8,121.2,123.6,125.2,125.9,127.5,128.1,129.0,130.6,133.8,138.0$, 158.6.

9-Amino-10-(3-methylphenyl)anthracene (9d). IR (KBr): $v_{\max } 3476,3386 \mathrm{~cm}^{-1}\left(\mathrm{NH}_{2}\right)$; UV $\left(\mathrm{CHCl}_{3}\right): \lambda_{\max } \mathrm{nm}(\log \varepsilon) 270$ (4.79), 380 (3.49), 420 (3.85); ${ }^{1} \mathrm{H}$ NMR (DMSO-d $\left.d_{6}\right): \delta 2.44(\mathrm{~s}$, $3 \mathrm{H}), 6.65(\mathrm{~s}, 2 \mathrm{H}), 7.10-7.11(\mathrm{~m}, 2 \mathrm{H}), 7.29-7.32(\mathrm{~m}, 5 \mathrm{H}), 7.40-7.44(\mathrm{~m}, 3 \mathrm{H}), 8.40(\mathrm{~m}, 2 \mathrm{H}) ;{ }^{13} \mathrm{C}$ NMR $\left(\mathrm{CDCl}_{3}\right): \delta 21.6,117.9,121.1,123.7,125.2,127.5,127.8,127.9,128.3,129.2,130.5$, $137.8,138.0,139.5$.

9-Amino-10-(naphth-1-yl)anthracene (9e). IR (KBr): $v_{\max }$ 3494, $3404 \mathrm{~cm}^{-1}\left(\mathrm{NH}_{2}\right)$ : UV $\left(\mathrm{CHCl}_{3}\right): \lambda_{\max }[\mathrm{nm}](\log \varepsilon) 270$ (4.87), 360, (3.59), 380 (3.85), 420 (3.890; ${ }^{1} \mathrm{H}$ NMR (DMSO$\left.d_{6}\right): \delta 6.60(\mathrm{~s}, 1 \mathrm{H}), 6.96-6.99(\mathrm{~m}, 2 \mathrm{H}), 7.18-7.24(\mathrm{~m}, 5 \mathrm{H}), 7.45-7.49(\mathrm{~m}, 4 \mathrm{H}), 8.40(\mathrm{~d}, J=8.8$ $\mathrm{Hz}, 2 \mathrm{H}$ ); ${ }^{13} \mathrm{C}$ NMR (DMSO- $\left.d_{6}\right): \delta 118.0,121.2,123.8,124.6,125.5,125.8,126.0,126.2$, $126.9,127.7,127.9,128.3,130.0,131.3,133.9,134.2,127.3,138.3$.

9-Amino-10-(3,4-dimethoxyphenyl)anthracene (9f). IR (KBr): $v_{\max } 3494,3401 \mathrm{~cm}^{-1}\left(\mathrm{NH}_{2}\right)$; UV $\left(\mathrm{CHCl}_{3}\right): \lambda_{\max }[\mathrm{nm}](\log \varepsilon) 272$ (4.93), 360 (3.52) 380 (3.84), 420 (3.87); ${ }^{1} \mathrm{H}$ NMR (DMSO-d D $_{6}: \delta 3.69$ (s, 3H), 3.85 (s, 3H), 6.63 (s, 2H), $6.81(\mathrm{dd}, J=8.0 \mathrm{~Hz}, 1 \mathrm{H}), 6.84(\mathrm{~s}, 1 \mathrm{H})$, $7.11(\mathrm{~d}, J=8.0 \mathrm{~Hz}, 1 \mathrm{H}), 7.26-7.29$ (m, 4H), 7.45-7.48 (m, 2H), 7.47, 8.37 (d, $J=8.4 \mathrm{~Hz}, 2 \mathrm{H}$ ); ${ }^{13} \mathrm{C}$ NMR (DMSO- $d_{6}$ ): $\delta 56.0,56.1,111.2,115.2,117.9,121.1,123.7,124.3,125.3,126.9$, $127.7,130.8,132.9,137.9,148.2,148.9$.

9-Amino-10-(pyrid-2-yl)anthracene (9g). IR (KBr) $v_{\max }$ 3493, $3401 \mathrm{~cm}^{-1}\left(\mathrm{NH}_{2}\right) ; \quad \mathrm{UV}$ $\left(\mathrm{CHCl}_{3}\right): \lambda_{\max }[\mathrm{nm}](\log \varepsilon) 270$ (4.91), 360 (3.51), 279 (3.88), 420 (3.91); ${ }^{1} \mathrm{H}$ NMR (DMSO$\left.d_{6}\right): \delta 6.60(\mathrm{~s}, 2 \mathrm{H}), 7.30-7.35(\mathrm{~m}, 4 \mathrm{H}), 7.45-7.46(\mathrm{~m}, 4 \mathrm{H}), 7.90-7.91(\mathrm{~m}, 1 \mathrm{H}), 8.35(\mathrm{~d}, J=8.8$ $\mathrm{Hz}, 2 \mathrm{H}), 8.80$ (br s, $1 \mathrm{H}) ;{ }^{13} \mathrm{C} \mathrm{NMR}\left(\mathrm{CDCl}_{3}\right.$ :) $\delta 117.7,121.3,122.0,123.5,125.1,125.7,125.9$, 126.6, 127.7, 130.5, 136.3, 139.1, 150.1, 159.2. Anal. Calcd for $\mathrm{C}_{19} \mathrm{H}_{14} \mathrm{~N}_{2}: \mathrm{C}, 84.42 ; 5.22 ; \mathrm{N}$, 10.36. Found: C, 84.49; H, 5.25; N, 10.42 .

9-Amino-10-(thien-3-yl)anthracene (9h). IR (KBr): $v_{\max }$ 3495, $3407 \mathrm{~cm}^{-1}\left(\mathrm{NH}_{2}\right)$; UV $\left(\mathrm{CHCl}_{3}\right): \lambda_{\max }[\mathrm{nm}](\log \varepsilon) 270$ (4.89), 361 (3.52), 379 (3.85), 420 (3.96): ${ }^{1} \mathrm{H}$ NMR (DMSO$\left.d_{6}\right): \delta 6.69(\mathrm{~s}, 2 \mathrm{H}), 7.11(\mathrm{~m}, 1 \mathrm{H}), 7.27-7.32(\mathrm{~m}, 4 \mathrm{H}), 7.49-7.52(\mathrm{~m}, 2 \mathrm{H}), 7.56-7.78(\mathrm{~m}, 1 \mathrm{H})$, $8.38(\mathrm{~d}, J=7.9 \mathrm{~Hz}, 2 \mathrm{H}) ;{ }^{13} \mathrm{C} \mathrm{NMR}\left(\mathrm{CDCl}_{3}\right) \delta 117.9,121.1,121.6,123.8,125.1,125.2,125.4$, $127.5,131.2,131.6,138.1,139.26$.

\section{General procedure for the preparation of $N$-acetyl derivatives 11a-f,h}

A $100 \mathrm{mg}$ sample of the appropriate 9-aminoanthracene 9 was added to $5 \mathrm{~mL}$ of acetic 
anhydride which was previously cooled to $0{ }^{\circ} \mathrm{C}$. Within $10 \mathrm{~min}$, crystals of the amide precipitated. The crystals were collected by vacuum filtration, washed first with water then cold acetone, and dried. The amides were sufficiently pure for analysis. The spectral data for 11a-f,h are given below.

$N$-Acetyl-9-amino-10-phenylanthracene (11a). IR (KBr): $v_{\max }: 3260,1658 \mathrm{~cm}^{-1}$; UV $\left(\mathrm{CHCl}_{3}\right): \lambda_{\max }[\mathrm{nm}](\log \varepsilon): 358$ (3.81), 376 (3.93), 396 (3.92) $\left(\mathrm{lit}^{5} 356\right.$ [3.81], 375, [3.93] 397 [3.92]); ${ }^{1} \mathrm{H} \mathrm{NMR}\left(\mathrm{CHCl}_{3}\right): \delta 2.37$ (s, 3H), 7.40-7.43 (m, 4H), 7.57-7.59 (m, 4H), 7.61-7.65 $(\mathrm{m}, 3 \mathrm{H}), 8.16(\mathrm{~d}, J=8.8 \mathrm{~Hz}, 2 \mathrm{H}), 10.27(\mathrm{~s}, 1 \mathrm{H}) ;{ }^{13} \mathrm{C} \mathrm{NMR}\left(\mathrm{CDCl}_{3}\right): \delta 23.4,124.7,126.2$, $126.9,128.4,128.5,129.2,130.2,130.6,130.7,131.4,136.4,128.5,170.4$.

$N$-Acetyl-9-amino-10-(4-methoxyphenyl)anthracene (11b). IR (KBr): $v_{\max } 3265,1658 \mathrm{~cm}^{-}$ 1; UV $\left(\mathrm{CHCl}_{3}\right): \lambda_{\max }[\mathrm{nm}](\log \varepsilon) 264$ (5.10), 342 (3.61), $360(3.85), 380$ (3.91), $397(3.93) ;{ }^{1} \mathrm{H}$ NMR (DMSO-d $\left.)_{6}\right): \delta 2.36(\mathrm{~s}, 3 \mathrm{H}), 3.88(\mathrm{~s}, 3 \mathrm{H}), 7.18,7.20\left(\mathrm{AA}^{\prime} 4-\mathrm{MeOC}_{6} \mathrm{H}_{4}\right), 7.31$ (BB'), $7.41(\mathrm{t}, J=7.2 \mathrm{~Hz}, 2 \mathrm{H}), 7.53(\mathrm{t}, J=7.2 \mathrm{~Hz}, 2 \mathrm{H}), 7.59$ (d, $J=8.5 \mathrm{~Hz}, 2 \mathrm{H}), 8.14$ (d, $J=8.5 \mathrm{~Hz}$, $2 \mathrm{H}), 10.24(\mathrm{~s}, 1 \mathrm{H}) ;{ }^{13} \mathrm{C} \mathrm{NMR}\left(\mathrm{DMSO}-d_{6}\right): \delta 23.4,55.7,114.6,124.7,126.1,126.2,127.0$, $128.4,130.3,130.4,130.5,133.5,136.3,159.3,170.1$.

$N$-Acetyl-9-amino-10-(2-methoxyphenyl)anthracene (11c). IR (KBr): $v_{\max } 3260,1665 \mathrm{~cm}^{-1}$; $\mathrm{UV}\left(\mathrm{CHCl}_{3}\right): \lambda_{\max }[\mathrm{nm}]$ (log $\left.\varepsilon\right) 262$ (4.98), 340 (3.61), 356 (3.82), 376 (3.97), $397(3.95) ;{ }^{1} \mathrm{H}$ NMR (DMSO-d $): \delta 2.36(\mathrm{~s}, 3 \mathrm{H}), 3.58(\mathrm{~s}, 3 \mathrm{H}), 7.18-7.20(\mathrm{~m}, 2 \mathrm{H}), 7.28(\mathrm{~d}, J=8.4 \mathrm{~Hz}, 2 \mathrm{H})$, $7.38(\mathrm{t}, J=8.4 \mathrm{~Hz}, 2 \mathrm{H}), 7.46(\mathrm{~m}, 2 \mathrm{H} 7.51(\mathrm{t}, J=8.4 \mathrm{~Hz}, 2 \mathrm{H}), 8.11(\mathrm{~d}, J=8.6 \mathrm{~Hz}, 2 \mathrm{H}), 10.20$ $(\mathrm{s}, 1 \mathrm{H}) ;{ }^{13} \mathrm{C}$ NMR $\left(\mathrm{DMSO}_{\mathrm{d}}\right): \delta 23.4,55.7,112.1,121.2,124.7,126.0,126.1,126.6,126.7$, $126.8,128.9,130.4,132.7,133.5,158.0,170.4$.

$N$-Acetyl-9-amino-10-(3-methylphenyl)anthracene (11d). IR (KBr): $v_{\max } 3209,1655 \mathrm{~cm}^{-1}$; $\mathrm{UV}\left(\mathrm{CHCl}_{3}\right): \lambda_{\max }[\mathrm{nm}](\log \varepsilon) 262$ (4.86), 342 (3.54), 358 (3.83), 376 (3.98), 398 (3.94); ${ }^{1} \mathrm{H}$ NMR (DMSO-d $): \delta 2.35(\mathrm{~s}, 3 \mathrm{H}), 2.47(\mathrm{~s}, 3 \mathrm{H}), 7.17-7.20(\mathrm{~m}, 2 \mathrm{H}), 7.39-7.41(\mathrm{~m}, 3 \mathrm{H}), 7.43(\mathrm{~s}$, 1H), 7.49-7.55 (m, 5H), $8.13(\mathrm{~d}, J=8.4 \mathrm{~Hz}, 2 \mathrm{H}), 10.24(\mathrm{~s}, 1 \mathrm{H}) ;{ }^{13} \mathrm{C}$ NMR (DMSO- $\left.d_{6}\right) \delta 21.6$, 23.4, 24.7, 126.1, 126.2, 127.0, 128.3, 128.5, 128.9, 129.0, 130.2, 130.5. 131.9, 136.6, 128.3, $138.5,170.3$.

$N$-Acetyl-9-amino-10-(naphth-1-yl)anthracene (11e). IR (KBr): $v_{\max } 3214,1650 \mathrm{~cm}^{-1}$; UV $\left(\mathrm{CHCl}_{3}\right): \lambda_{\max }[\mathrm{nm}]$ (log ع): 262 (4.96), 340 (3.56), 357 (3.86), 376 (4.02), $398(4.01) ;{ }^{1} \mathrm{H}$ NMR (DMSO-d $): \delta 6.82-6.84(\mathrm{~m}, 1 \mathrm{H}), 7.23(\mathrm{~d}, J=8.0 \mathrm{~Hz}, 2 \mathrm{H}), 7.29-7.33(\mathrm{~m}, 3 \mathrm{H}), 7.48-$ $7.54(\mathrm{~m}, 4 \mathrm{H}, 7.56(\mathrm{t}, J=7.9 \mathrm{~Hz}, 1 \mathrm{H}), 7.66(\mathrm{t}, J=7.9 \mathrm{~Hz}, 1 \mathrm{H}), 8.08-8.20(\mathrm{~m}, 4 \mathrm{H}), 10.30$ (s, 
$1 \mathrm{H}) ;{ }^{13} \mathrm{C}$ NMR (DMSO-d $): \delta 124.8,126.1,126.2,126.4,126.7,127.1,128.4,128.8,129.0$, $129.5,130.9,131.0,133.3,133.9,134.1,136.0,170.4$.

$N$-Acetyl-9-amino-10-(3,4-dimethoxyphenyl)anthracene (11f). IR (KBr): $v_{\max } 3214$ (HN), $1671 \mathrm{~cm}^{-1}$ (CO); UV $\left(\mathrm{CHCl}_{3}\right): \lambda_{\max }[\mathrm{nm}](\log \varepsilon) 262$ (4.90), 342 (3.60), 354 (3.86), 376 (4.02), 398 (4.00); ${ }^{1} \mathrm{H}$ NMR (DMSO-d $): \delta 2.34(\mathrm{~s}, 3 \mathrm{H}), 3.71(\mathrm{~s}, 3 \mathrm{H}), 3.86(\mathrm{~s}, 3 \mathrm{H}), 6.90(\mathrm{~d}, J=8.0$ $\mathrm{Hz}, 2 \mathrm{H}), 6.97$ (s, 1H), 7.19 ((d, J=8.0 Hz, 1H), 7.42 (t, $J=8.9 \mathrm{~Hz}, 2 \mathrm{H}), 7.53$ (t, $J=8.0 \mathrm{~Hz}$, 2H), $7.62(\mathrm{~d}, J=8.8 \mathrm{~Hz}, 2 \mathrm{H}), 8.12$ (d, $J=8.8 \mathrm{~Hz}, 2 \mathrm{H}), 10.23$ (s, 1H); ${ }^{13} \mathrm{C}$ NMR (DMSO-d 6 ): $\delta 23.4,56.2,56.3,112.5,115.1,123.6,124.6,125.9,126.1,127.2,128.4,130.3,130.8,136.5$, $148.9,149.3,170.3$.

$N$-Acetyl-9-amino-10-(thien-3-yl)anthracene (11h). IR (KBr): $v_{\max } 3261(\mathrm{HN}), 1665 \mathrm{~cm}^{-1}$ (CO); UV ( $\left.\mathrm{CHCl}_{3}\right): \lambda_{\max }[\mathrm{nm}]$ (log $\left.\varepsilon\right): 260$ (4.98), 342 (3.54), 354 (3.86), 376 (4.01), 398 (3.98); ${ }^{1} \mathrm{H}$ NMR (DMSO-d $\left.)_{6}\right): \delta 7.24-7.25(\mathrm{~m}, 1 \mathrm{H}), 7.45(\mathrm{t}, J=7.45 \mathrm{~Hz}, 2 \mathrm{H}), 7.54(\mathrm{t}, J=8.4$ $\mathrm{Hz}, 2 \mathrm{H}), 7.56(\mathrm{~s}, 1 \mathrm{H}), 7.67$ (d, $J=8.4 \mathrm{~Hz}, 2 \mathrm{H}), 7.86-7.88(\mathrm{~m}, 1 \mathrm{H}), 8.14(\mathrm{~d}, J=8.8 \mathrm{~Hz}, 2 \mathrm{H})$, $10.30(\mathrm{~s}, 1 \mathrm{H}) ;{ }^{13} \mathrm{C}$ NMR (DMSO-d 6 ): $\delta 23.4,124.7,126.0,126.1,126.3,126.9,127.2,128.4$, $130.8,131.2,131.6,138.1,170.3$.

General procedure for the preparation of peri-substituted 10-aryl-9-aminoanthracenes (9i-s) and 10-aryl-9,10-dihydroanthracen-9-imines (8a-f)

The reactions were carried out in similar manner as those described for 9-aminoanthracenes 9a-h with the exception that the appropriate 2-arylmethylbenzonitrile 6 (10 mmol) and the bromoarene 1 (20 mmol) were added sequentially to a solution containing LDA (50 mmol) in THF (10 mL). The spectral data of the products 9i-s, 8a-f are given below.

9-Amino-1-methoxy-10-phenylanthracene (9i). IR (KBr): $v_{\max } 3488,3401 \mathrm{~cm}^{-1}\left(\mathrm{NH}_{2}\right)$; UV $\left(\mathrm{CHCl}_{3}\right) ; \lambda_{\max }[\mathrm{nm}](\log \varepsilon): 270$ (4.98), 370 (3.86), 388 (4.01), 437 (3.82); ${ }^{1} \mathrm{H}$ NMR (DMSO$\left.d_{6}\right): \delta 4.00(\mathrm{~s}, 3 \mathrm{H}), 6.63(\mathrm{~d}, J=7.3 \mathrm{~Hz}, 1 \mathrm{H}), 6.88(\mathrm{~d}, J=8.4 \mathrm{~Hz}, 1 \mathrm{H}), 7.07$ (t,J=7.6 Hz, 1H), 7.25-7.30, (m,6H), 7.43-7.45 (m, 1H), $7.54(\mathrm{t}, \mathrm{J}=7.0 \mathrm{~Hz}, 2 \mathrm{H}), 8.32-8.35(\mathrm{~m}, 1 \mathrm{H})$; ${ }^{13} \mathrm{C} \mathrm{NMR}$ $\left(\mathrm{DMSO}-d_{6}\right): \delta 56.4,101.3,109.1,117.5,119.5,121.9,122.5,123.5,125.7,126.3,126.5$, $127.4,129.2,131.3,132.3,133.1,140.6,143.6,158.9$.

9-Amino-1-methoxy-10-(4-methoxyphenyl)anthracene (9j). IR (KBr): $v_{\max } 3488,3401 \mathrm{~cm}^{-}$

${ }^{1}\left(\mathrm{NH}_{2}\right): \mathrm{UV}\left(\mathrm{CHCl}_{3}\right): \lambda_{\max }[\mathrm{nm}](\log \varepsilon) 270$ (4.93), $370(3.84), 388$ (4.02), $437(3.83) ;{ }^{1} \mathrm{H}$ NMR (DMSO-d $\left.)_{6}\right): \delta 3.67(\mathrm{~s}, 3 \mathrm{H}), 4.00(\mathrm{~s}, 3 \mathrm{H}), 6.63(\mathrm{~d}, J=7.3 \mathrm{~Hz}, 1 \mathrm{H}), 6.88(\mathrm{~d}, J=8.4 \mathrm{~Hz}$, 
1H), 7.01, 7.03 (AA' 4-MeOC $\left.6 \mathrm{H}_{4}\right)$, , 7.12, $7.14\left(\mathrm{BB}^{\prime}\right),(\mathrm{d}, J=7.6 \mathrm{~Hz}, 2 \mathrm{H}), 7.13$, (d, J = 7.6 Hz, 1H), 7.40-7.45 (m, 1H), $7.54(\mathrm{~m}, 3 \mathrm{H}), 7.55(\mathrm{~d}, J=7.2 \mathrm{~Hz}, 1 \mathrm{H}), 8.32(\mathrm{~d}, J=7.6 \mathrm{~Hz}, 1 \mathrm{H}) ;{ }^{13} \mathrm{C}$ NMR (DMSO- $\left.d_{6}\right): \delta 55.6,56.4,101.3,109.2,114.6,117.6,119.7,121.6,122.5,123.5,125.6$, 126.4, 131.2, 131.6, 132.5, 133.3, 133.5, 143.4, 158.7, 158.9.

9-Amino-1-methoxy-10-(3-methylphenyl)anthracene (9k). ${ }^{1} \mathrm{H}$ NMR (acetone- $\left.d_{6}\right): \delta 2.16$ (s, 3H), 4.00 (s, 3H), $6.92(\mathrm{t}, J=8.8 \mathrm{~Hz}, 2 \mathrm{H}), 7.00$ (t, $J=8.0 \mathrm{~Hz}, 2 \mathrm{H}), 7.08$ (t, $J=8.8 \mathrm{~Hz}, 1 \mathrm{H})$, $7.43(\mathrm{~m}, 4 \mathrm{H}), 7.66(\mathrm{~d}, J=8.0 \mathrm{~Hz}, 1 \mathrm{H}), 8.33(\mathrm{~d}, J=8.0 \mathrm{~Hz}, 1 \mathrm{H})$.

9-Amino-1-methoxy-10-(naphth-1-yl)anthracene (9l). ${ }^{1} \mathrm{H}$ NMR (DMSO-d ${ }_{6}$ ): $\delta 4.10$ (s, 3H), $6.68(\mathrm{~d}, J=7.4 \mathrm{~Hz}, 1 \mathrm{H}), 6.72(\mathrm{~d}, J=8.4 \mathrm{~Hz}, 1 \mathrm{H}), 6.98$ (t, $J=8.4 \mathrm{~Hz}, 1 \mathrm{H}), 7.09$ (t, $J=8.0 \mathrm{~Hz}$, $3 \mathrm{H}), 7.20(\mathrm{~m} .3 \mathrm{H}), 7.25(\mathrm{t}, J=7.4 \mathrm{~Hz}, 1 \mathrm{H}), 7.46(\mathrm{~m}, 2 \mathrm{H}), 7.70(\mathrm{t}, J=8.0 \mathrm{~Hz}, 1 \mathrm{H}), 8.04(\mathrm{t}, J=$ $8.4 \mathrm{~Hz}, 2 \mathrm{H}), 8.33(\mathrm{~d}, J=8.0 \mathrm{~Hz}, 1 \mathrm{H})$.

9-Amino-1,8-dimethoxy-10-phenylanthracene (9m). IR (KBr): $v_{\max }: 3489,3396 \mathrm{~cm}^{-1}$ $\left(\mathrm{NH}_{2}\right) ; \mathrm{UV}\left(\mathrm{CHCl}_{3}\right): \lambda_{\max }[\mathrm{nm}]$ (log $\left.\varepsilon\right) 260$ (4.98), 380 (3.86), 399 (4.16), $446(3.80) ;{ }^{1} \mathrm{H}$ NMR (DMSO-d $)_{6}$ ): $\delta 3.98$ (s, 6H), $6.62(\mathrm{~d}, J=7.2 \mathrm{~Hz}, 2 \mathrm{H}), 6.74$ (d, $\left.J=8.4 \mathrm{~Hz}, 2 \mathrm{H}\right), 7.07$ (t, $J$ $=8.4 \mathrm{~Hz}, 2 \mathrm{H}), 7.19(\mathrm{~d}, J=7.2 \mathrm{~Hz}, 2 \mathrm{H}), 7.43-7.45(\mathrm{~m}, 1 \mathrm{H}), 7.51(\mathrm{t}, J=7.2 \mathrm{~Hz}, 2 \mathrm{H}), 8.11(\mathrm{~s}$, $2 \mathrm{H}$ ); [exchangeable with $\mathrm{D}_{2} \mathrm{O}$ ). ${ }^{13} \mathrm{C}$ NMR (DMSO- $\left.d_{6}\right): \delta 56.5,101.5,109.1,119.2,120.3$, $126.3,127.3,129.3,132.3,133.7,141.4,146.3,159.2$.

9-Amino-1,8-dimethoxy-10-(napth-1-yl)anthracene (9n). ${ }^{1} \mathrm{H}$ NMR (acetone- $\left.d_{6}\right): \delta 4.06$ (s, $6 \mathrm{H}), 6.59(\mathrm{~d}, J=8.4 \mathrm{~Hz}, 2 \mathrm{H}), 6.94(\mathrm{~d}, J=7.5 \mathrm{~Hz}, 1 \mathrm{H}), 6.98(\mathrm{t}, J=8.4 \mathrm{~Hz}, 2 \mathrm{H}), 7.11(\mathrm{~d}, J=$ $8.0 \mathrm{~Hz}, 1 \mathrm{H}), 7.22(\mathrm{t}, J=7.5 \mathrm{~Hz}, 1 \mathrm{H}), 7.40(\mathrm{~d}, J=7.5 \mathrm{~Hz}, 1 \mathrm{H}), 7.48(\mathrm{t}, J=8.0 \mathrm{~Hz}, 1 \mathrm{H}), 7.69(J$ $=7.5 \mathrm{~Hz}, 1 \mathrm{H}), 8.04\left(\mathrm{t}, J^{1}=8.0 \mathrm{~Hz}, J^{2} 7.5 \mathrm{~Hz}, 2 \mathrm{H}\right), 8.26(\mathrm{~s}, 2 \mathrm{H})$.

9-Amino-1-methoxy-4-methyl-10-phenylanthracene (9o). UV $\left(\mathrm{CHCl}_{3}\right): \lambda_{\max }[\mathrm{nm}](\log \varepsilon)$ 246 (4.93), 273 (6.97), 374 3.62), 392 (3.82), 432 (3.64); ${ }^{1} \mathrm{H}$ NMR (acetone- $\left.d_{6}\right): \delta 1.78$ (s, $3 \mathrm{H}), 4.00(\mathrm{~s}, 3 \mathrm{H}), 6.55(\mathrm{~d}, J=7.9 \mathrm{~Hz}, 1 \mathrm{H}), 6.90(\mathrm{~d}, J=8.8 \mathrm{~Hz}, 1 \mathrm{H}), 7.22-7.25(\mathrm{~m}, 1 \mathrm{H}), 7.28-$ $7.29(\mathrm{~m}, 5 \mathrm{H}), 7.41-7.43(\mathrm{~m}, 2 \mathrm{H}), 8.25(\mathrm{~d}, J=8.0 \mathrm{~Hz}, 1 \mathrm{H})$.

9-Amino-1-methoxy-4-methyl-10-(4-methoxyphenyl)anthracene (9p). UV ( $\left.\mathrm{CHCl}_{3}\right): \lambda_{\max }$ [nm] (log \&) 242 (4.40), 270 (4.26), 375 (3.61), 393, (3.90) 440 (3.75); ${ }^{1} \mathrm{H}$ NMR (DMSO-d ${ }_{6}$ ): $\delta 1.74$ (s, 3H), 3.82 (s, 3H), 3.96 (s, 3H), 6.53 (d, $J=7.4 \mathrm{~Hz}, 1 \mathrm{H}), 6.90$ (d, $J=7.4 \mathrm{~Hz}, 1 \mathrm{H})$, 6.98, 7.01 (AA' 4-MeOC $\mathrm{H}_{4}$ ), 7.13, 7.15 (BB') 7.25-7.32 (m, 5H), 8.29 (d, $\left.J=7.6 \mathrm{~Hz}, 1 \mathrm{H}\right)$. ${ }^{13} \mathrm{C}$ NMR (DMSO- $d_{6}$ ): $\delta 26.4,55.6,56.3,101.2,110.1,113.5,117.7,121.9,122.7,123.1$, 126.3, 126.7, 126.9, 129.4, 132.8, 132.9, 133.8, 135.4, 143.8, 157.7, 158.6.

9-Amino-1-methoxy-4-methyl-10-(3-methylphenyl)anthracene (9q). ${ }^{1} \mathrm{H}$ NMR (DMSO- $\left.d_{6}\right)$ : 
$\delta 1.75(\mathrm{~s}, 3 \mathrm{H}), 3.60(\mathrm{~s}, 3 \mathrm{H}), 3.96(\mathrm{~s}, 3 \mathrm{H}), 6.54(\mathrm{~d}, J=7.6 \mathrm{~Hz}, 1 \mathrm{H}), 6.94(\mathrm{~d}, J=7.6 \mathrm{~Hz}, 1 \mathrm{H})$, 6.99 (d, $J=7.6 \mathrm{~Hz}, 1 \mathrm{H}), 7.09$ (t, $J=7.6 \mathrm{~Hz}, 1 \mathrm{H}), 7.20$ (m, 3H), 7.28 (m, 4H), 8.34 (d, $J=7.6$ $\mathrm{Hz}, 1 \mathrm{H})$.

9-Amino-1-methoxy-4-methyl-10-(naphth-1-yl)anthracene (9r). ${ }^{1} \mathrm{H}$ NMR (acetone- $\left.d_{6}\right)$ : $\delta$ 1.52 (s, 3H), 4.08 (s, 3H), $6.62(\mathrm{~d}, J=7.8 \mathrm{~Hz}, 1 \mathrm{H}), 6.91$ (d, $J=7.8 \mathrm{~Hz}, 1 \mathrm{H}), 6.98(\mathrm{~d}, J=7.8$ Hz, 1H), 7.10 (m. 3H), 7.26 (m, 3H), 7.41 (d, J=7.6 Hz, 1H), 7.45 (t, J= 7.6 Hz, 1H), 7.60 (t, $J=8.0 \mathrm{~Hz}, 1 \mathrm{H}), 8.03$ (t, $J=8.0 \mathrm{~Hz}, 2 \mathrm{H}), 8.29$ (d, $J=8.0 \mathrm{~Hz}, 1 \mathrm{H})$.

9-Amino-1-methoxy-(3,4,5-trimethoxyphenyl)-4-methylanthracene (9s). UV ( $\left.\mathrm{CHCl}_{3}\right)$ : $\lambda_{\max }[\mathrm{nm}](\log \varepsilon) 271$ (4.55), 373 (3.54), 391 (3.94), 436 (3.76); ${ }^{1} \mathrm{H}$ NMR (DMSO- $\left.d_{6}\right): \delta 1.90$ (s, 3H), 3.70 (s, 6H), 3.77 (s, 3H), 4.00 (s, 3H), 6.54 (s, 2H), 6.57 (d, J=8.8 Hz, 1H), 6.95 (d, $J=8.0 \mathrm{~Hz}, 1 \mathrm{H}), 7.29(\mathrm{~m}, 5 \mathrm{H}), 8.29(\mathrm{~d}, J=8.8 \mathrm{~Hz}, 1 \mathrm{H})$.

\section{Acknowledgements}

This work was support, in part, by grants from the Welch Foundation, Houston, TX, and the Petroleum Research Corporation, administered by the American Chemical Society.

\section{References}

1. Pansegrau, P. D.; Rieker, W. F.; Meyers, A. I. J. Am. Chem. Soc.1988, 110, 7148.

2. Khanapure, S. P.; Biehl, E. R. Acc. Chem. Res. 1989, 22, 275.

3. Roberts, J. D.; Semenow, D.; Simmons, H. E.; Carlsmith, L. A. J. Am. Chem. Soc.1956, $78,601$.

4. Dutt, M.; Fravel, B.; Ford, G.P.; Biehl, E. R. J. Org. Chem. 1994, 59, 497.

5. Rigaudy, J.; Cauquis, G.; Izoret, G.; Baranne-LaFont, J. J. Chem. Soc. 1961, 1842.

6. Barry-Barnett, E. D.; Cook, J. W. J. Chem. Soc. 1923, 128, 2631

7. Wang, A.; Maguire, J. A.; Biehl, E. J. Org. Chem. 1998, 63, 2451.

8. Crenshaw, L.; Khanapure, S. P.; Biehl, E. R. J. Org. Chem. 1988, 29, 3777.

9. Wang, A.; Zhang, H.; Biehl, E. R. Heterocycles 2000, 50, 300.

10. Roberts, J. D.; Vaughan, C. W.; Carlsmith, L. A.; Semenov, D. A. J. Am. Chem. 
Soc.1956, 78, 611.

11. Johnson, C. K. 'ORTEP, Report ORNL-3794', Oak Ridge National Laboratory, Oak Ridge, Tennessee, 1965. 